\title{
Universiteit
}

Leiden

The Netherlands

\section{Assessment of Appropriateness of Screening Community-Dwelling Older People to Prevent Functional Decline}

Drewes, Y.M.; Gussekloo, J.; Meer, V. van der; Rigter, H.; Dekker, J.H.; Goumans, M.J.B.M.; ... ; Assendelft, W.J.J.

\section{Citation}

Drewes, Y. M., Gussekloo, J., Meer, V. van der, Rigter, H., Dekker, J. H., Goumans, M. J. B. M., ... Assendelft, W. J. J. (2012). Assessment of Appropriateness of Screening CommunityDwelling Older People to Prevent Functional Decline. Journal Of The American Geriatrics Society, 60(1), 42-50. doi:10.1111/j.1532-5415.2011.03775.x

Version: $\quad$ Not Applicable (or Unknown)

License: $\quad$ Leiden University Non-exclusive license

Downloaded from: https://hdl.handle.net/1887/117472

Note: To cite this publication please use the final published version (if applicable). 


\section{Assessment of Appropriateness of Screening Community- Dwelling Older People to Prevent Functional Decline}

Yvonne M. Drewes, LLM, MD, ${ }^{a}$ Jacobijn Gussekloo, MD, PhD, ${ }^{a}$ Victor van der Meer, MD, PhD, ${ }^{a}$ Henk Rigter, PhD, ${ }^{c}$ Janny H. Dekker, MD, PhD, ${ }^{d}$ Marleen J. B. M. Goumans, PhD, ${ }^{e}$ Job F. M. Metsemakers, MD, PhD, ${ }^{f}$ Riki van Overbeek, ${ }^{g}$ Sophia E. de Rooij, MD, PhD, ${ }^{b}$ Henk J. Schers, MD, PhD, ${ }^{i}$ Marieke J. Schuurmans, PhD, RN, ${ }^{j}$ Ferd Sturmans, MD, PhD, ${ }^{c}$ Kerst de Vries, MD, ${ }^{k}$ Rudi G. J. Westendorp, MD, PhD, ${ }^{b}$ Annet W. Wind, MD, PhD, ${ }^{a}$ and Willem J. J. Assendelft, $\mathrm{MD}, \mathrm{PhD}^{a}$

OBJECTIVES: To identify appropriate screening conditions, stratified according to age and vulnerability, to prevent functional decline in older people.

DESIGN: A RAND/University of California at Los Angeles appropriateness method.

SETTING: The Netherlands.

PARTICIPANTS: A multidisciplinary panel of 11 experts. MEASUREMENTS: The panelists assessed the appropriateness of screening for 29 conditions mentioned in guidelines from four countries, stratified according to age $(60-74,75-84, \geq 85)$ and health status (general, vital, and vulnerable) and received a literature overview for each condition, including the guidelines and up-to-date literature. After an individual rating round, panelists discussed disagreements and performed a second individual rating. The median of the second ratings defined the appropriateness of screening.

RESULTS: The panel rated screening to be appropriate in three of the 29 conditions, indicating that screening was expected to prevent functional decline. Screening for insuf-

From the ${ }^{a}$ Departments of Public Health and Primary Care, ${ }^{b}$ Gerontology and Geriatrics, Leiden University Medical Center, Leiden, ${ }^{c}$ Department of Public Health, Erasmus Medical Center, Rotterdam, ${ }^{\mathrm{d} D e p a r t m e n t}$ of General Practice, University Medical Center Groningen, University of Groningen, Groningen, ${ }^{\mathrm{e}}$ Institute for Research and Innovation, Centre of Expertise Innovations in Care, Rotterdam University, Rotterdam, ${ }^{\mathrm{f}}$ Department of General Practice, Maastricht University, Maastricht, ${ }^{g}$ Vilans, Dutch Knowledge Centre on Ageing, Utrecht, ${ }^{\text {h Section of }}$ Geriatric Medicine, Department of Internal Medicine, Academic Medical Center, University of Amsterdam, Amsterdam, ${ }^{\mathrm{i}}$ Department of Primary and Community Care, Radboud University Nijmegen Medical Centre, Nijmegen, ${ }^{j}$ Department of Nursing Science, University Medical Center Utrecht, Utrecht, and ${ }^{\mathrm{k}}$ Department of Nursing Home Medicine, VU University Medical Center, Amsterdam, the Netherlands.

Address correspondence to Yvonne M. Drewes, Department of Public Health and Primary Care (Postzone V0-P), Leiden University Medical Center, PO Box 9600, 2300 RC Leiden, the Netherlands.

E-mail: y.m.drewes@lumc.nl

DOI: $10.1111 / \mathrm{j} .1532-5415.2011 .03775 . \mathrm{x}$ ficient physical activity was considered appropriate for all three age and health groups. Screening for cardiovascular risk factors and smoking was considered appropriate for the general and vital population aged 60 to 74 . Of the 261 ratings, $63(24 \%)$ were classified as uncertain, of which 42 $(67 \%)$ concerned the vulnerable population. The panelists considered conditions inappropriate mainly because of lack of an adequate screening tool or lack of evidence of effective interventions for positive screened persons.

CONCLUSION: The expert panel considered screening older people to prevent functional decline appropriate for insufficient physical activity and smoking and cardiovascular risk in specific groups. For other conditions, sufficient evidence does not support screening. Based on their experience, panelists expected benefit from developing tests and interventions, especially for vulnerable older people. J Am Geriatr Soc 60:42-50, 2012.

Key words: screening; elderly; primary care; public health

$\mathrm{T}$ he interest in screening community-dwelling older people is increasing, ${ }^{1-4}$ and several guidelines for such screening have been issued. ${ }^{5-13}$ Screening is a strategy used in a population to detect a disease, risk factor, or ailment in individuals with unrecognized signs or symptoms. In general, the intention of screening is to identify the screened condition early, enabling earlier intervention and management to postpone diseases and death, but older people (especially frail older people) do not always benefit from screening because of their shorter natural life expectancy and their lack of physiological reserve to tolerate the invasive interventions called for after screening. ${ }^{4}$ 


\begin{tabular}{ll}
\hline Table 1. Wilson and Jungner Criteria for Screening \\
\hline 1 & The condition sought should be an important health problem \\
2 & $\begin{array}{l}\text { There should be an accepted treatment for patients } \\
\text { with recognized disease }\end{array}$ \\
3 & Facilities for diagnosis and treatment should be available \\
4 & There should be a recognizable latent or early symptomatic stage \\
5 & There should be a suitable test or examination \\
6 & The test should be acceptable to the population \\
7 & $\begin{array}{l}\text { The natural history of the condition, including development from } \\
\text { latent to declared disease, should be adequately understood }\end{array}$ \\
8 & $\begin{array}{l}\text { There should be an agreed policy on whom to treat as patients } \\
9\end{array}$ \\
$\begin{array}{l}\text { The cost of case-finding (including diagnosis and treatment of } \\
\text { patients diagnosed) should be economically balanced in relation } \\
\text { to possible expenditure on medical care as a whole }\end{array}$ \\
10 & $\begin{array}{l}\text { Case-finding should be a continuing process and not a "once and } \\
\text { for all" project }\end{array}$ \\
\hline
\end{tabular}

For these older populations, screening can have an additional aim. In this age group, the aim is also to contribute to healthy aging, which is a prominent theme in current health policy. ${ }^{14-17}$ Healthy aging is not only a matter of maintaining good physical and mental health, but also of older people remaining independent and participating in social activities. As the general health status of older people declines, values such as functioning in daily life and well-being become more important than life expectancy. ${ }^{18}$ Therefore, it was postulated that a screening approach to community-dwelling older people would be appropriate if it aimed at preventing and postponing functional decline, ${ }^{19}$ but current screening guidelines tend to ignore this aim. In addition, specific research on screening in older people is scarce. Therefore, screening guidelines often have to address a lack of age-specific evidence.

In the present study, an expert panel assessed the contribution of screening of community-dwelling older people to the prevention of functional decline using the RAND/ University of California at Los Angeles (UCLA) appropriateness method. ${ }^{20-22}$ This method was chosen because a preceding literature search showed that the available scientific evidence was inconclusive. This RAND/UCLA appropriateness method was specifically developed to combine the available scientific evidence with the collective judgment of experts. To select conditions for this study, the content of general guidelines and protocols on screening and prevention was used. The appropriateness of screening the older population to prevent functional decline was assessed for several conditions by applying the most frequently used criteria for screening of this older population, formulated in 1968 by Wilson and Jungner (Table 1). ${ }^{23}$ Because the older population is heterogeneous, and it was hypothesized that age and vulnerability would be important determinants in assessing appropriateness, the present study stratified according to age ${ }^{24}$ and vulnerability. ${ }^{25,26}$

\section{METHODS}

The RAND/UCLA appropriateness method was used. ${ }^{20,22}$ The method was designed in the mid-1980s, primarily as an instrument to enable measurement of the overuse and underuse of medical and surgical procedures. Since then, this method has been used for many topics and its validity and reliability have been demonstrated in a wide variety of medical and preventive procedures that lack a firm evidence base. ${ }^{27-29}$ For a detailed description see Appendix S1.

\section{Selection of Screening Conditions and Literature Review}

Guidelines and protocols on screening and prevention were used to select conditions for this study. Conditions were selected from three Dutch guidelines and protocols on screening and prevention ${ }^{5-7}$ and from English-language guidelines of five leading healthcare institutes in the United States, Australia, and Great Britain. ${ }^{8-13}$ Two of these documents were specifically developed for vulnerable older people, ${ }^{6,10}$ but none of them was specifically aimed at prevention of functional decline.

A screening condition was considered eligible if it was recommended in one or more of these guidelines; this resulted in 29 conditions. To compile an overview of the evidence for each of these conditions, the guidelines and the literature references on which these guidelines were based were collected. For each condition separately, a scientist with expertise in the content of that condition was asked to comment on the guidelines and reference lists and to add up-to-date information if available. These files, one for each condition, formed the evidence package for the expert panel. The panelists used the literature overview from the evidence packages and their expertise to weigh the evidence for screening of each condition.

To acquire an overview of the differences between the guidelines and protocols, two researchers (YD, VvdM) independently divided the screening recommendations of the guidelines into the following groups: positive advice for older people in general, positive advice for specific groups of older people (people at risk, as defined in the guidelines), negative advice, insufficient evidence to give advice, or screening not mentioned in the guideline. Any disagreement between the two researchers was settled by consensus discussions or by a third party (JG).

\section{Expert Panel and Rating Process}

For the panel, 11 experts from disciplines involved in geriatric care and screening were recruited from eight university medical centers: seven physicians with scientific expertise, of whom four were general practitioners (JD, JM, HS, AW), two were clinical geriatricians (SdR, RW), and one was a nursing home physician $(\mathrm{KdV})$; three scientists, of whom two were public health scientists (MG, FS), and one was a nursing scientist (MS); and an expert from Vilans, a Dutch Knowledge Centre on Ageing ( $\mathrm{RvO})$. In brief, the RAND/UCLA appropriateness method entails two rounds of independent ratings by panelists, with one face-to-face group discussion (supervised by an independent chairman) between these rounds. ${ }^{22}$ The panelists rated the appropriateness of screening for each condition. The score of each panelist was equally weighed in the final ratings. One month before the meeting, panelists received the evidence packages, definitions of the terms used for the procedure, the criteria of Wilson and Jungner, ${ }^{23}$ and the rating sheets. 
In accordance with the RAND/UCLA appropriateness method, the expert panel was instructed to weigh evidence and to use their expert opinion for assessment of the contribution of screening to the prevention or postponement of functional decline for each specific condition. The panelists followed the previously developed criteria of Wilson and Jungner for each condition; they investigated whether evidence was present to fulfill the criteria for a specific condition, taking their expert opinion about a potential benefit into account.

Prevention or postponement of functional decline was defined as supporting the ability of older people to function as independently as possible. ${ }^{18}$ Screening was considered appropriate if the health benefits exceed the health risks by a margin that was sufficiently wide to make the procedure worth doing. ${ }^{20,22,30}$ The expert panel was asked to rate each condition for each of the three age groups $(60$ $-74,75-84, \geq 85)$ and for each of the three levels of health status (general, vital, and vulnerable).

In the present study, the general population was defined as the overall older population. This population was split into a vulnerable population with a high prevalence of diseases and disorders, a poorer prognosis, disability of various kinds, multiple problems simultaneously, and a vital population that was defined as nonvulnerable.

The rating process resulted in nine ratings per condition. Rating was done on a 1- to 9-point Likert scale ( 1 = extremely inappropriate, $5=$ uncertain or equivocal, and $9=$ extremely appropriate to screen).

The rating sheets were returned by mail and tabulated, and the results of the first-round rating were used to guide a subsequent 2-day face-to-face meeting of all panelists in March 2009. At the face-to-face meeting, headed by a moderator experienced in the RAND/UCLA appropriateness method (HR), ${ }^{6,31}$ each panelist received a report of his or her own first-round ratings, a frequency distribution, and the median of the whole panel. The individual ratings were blinded to other group members. Every condition was discussed to identify areas of disagreement, to highlight evidence not cited in the literature reviews, and to clarify specific definitions or wording of the conditions. In addition, panelists could revise existing conditions to better fit their judgment and could propose new conditions. The Wilson and Jungner criteria were used as leading principles in the discussion. After these discussions, in which the assessment was based on the combination of evidence and expert opinion, each panelist rerated all of the conditions on the 1- to 9-point scale. The entire discussion was audiotaped, and two researchers (YD, JG) made field notes. After the session, a report was written and sent to the panelists for their comments. These documents were used in the analysis to explain the outcomes of the ratings.

\section{Appropriateness}

The final appropriateness judgments were based on the median panel rating and level of disagreement for each condition in the second round, using the following definitions: all conditions with a median rating of 7 to 9 , rated without disagreement, were classified as appropriate; those with a median rating of 1 to 3 , rated without disagreement, were classified as inappropriate; and those with a median rating of 4 to 6 , as well as all conditions rated with disagreement, regardless of the median, were classified as uncertain. A condition was considered to be rated with disagreement when at least three panelists rated it in the 1 to 3 range, and at least three panelists rated it in the 7 to 9 range. ${ }^{32}$

\section{RESULTS}

\section{Recommendations by Guidelines}

The guidelines for screening ${ }^{5-13}$ showed a great variety of conditions and screening advice. None of the individual 29 conditions was addressed in all screening guidelines. The most frequently advised screening was for smoking status, followed by cardiovascular risk factors, malnutrition, and overweight. For abdominal aortic aneurysm, cognitive impairment, depression and anxiety, diabetes mellitus, and osteoporosis, the guidelines gave conflicting recommendations; some advised screening for these conditions, whereas others warned against screening. Table 2 gives an overview of the recommendations in the guidelines; the conditions included in the second rating process are also shown.

\section{RAND/UCLA appropriateness method}

In the first round with 29 conditions, there was disagreement in $23 \%(59 / 261)$ of the ratings. In the second round, after the face-to-face meeting, the disagreement was reduced to $3.4 \%$ (9/261). During the discussion sessions, three conditions were dropped because they were too difficult to define in an unequivocal way (social well-being, social support, and spare time), two conditions were divided into two parts (nutrition into malnutrition and undernutrition and burden of the informal caregiver into burden of the screened person as informal caregiver and burden of the informal caregivers around the screened person). One specification of a subgroup was added to abdominal aortic aneurysm and was discussed separately (abdominal aortic aneurysm in (ex-)smoking men). As a result, the second round also addressed 29 conditions.

\section{Appropriateness}

For the older population in general, screening for insufficient physical activity for all three age groups and screening for cardiovascular risk and smoking for aged 60 to 74 were rated appropriate, indicating that screening was expected to prevent functional decline (Table 3). Screening was rated uncertain for hearing impairment (all three age groups), colorectal cancer (60-74 and 75-84), the burden of the screened person as informal caregiver, smoking status $(75-84$ and $\geq 85)$, cardiovascular risk factors (75-84) and abdominal aortic aneurysm in (ex-)smoking men (6074), indicating serious doubts. For all the other conditions, screening of the general older population was considered inappropriate.

\section{Influence of Vulnerability}

Screening for insufficient physical activity was considered appropriate for all older persons (Table 3). Cardiovascular 
Table 2. Screening Conditions for Older Persons in the Guidelines

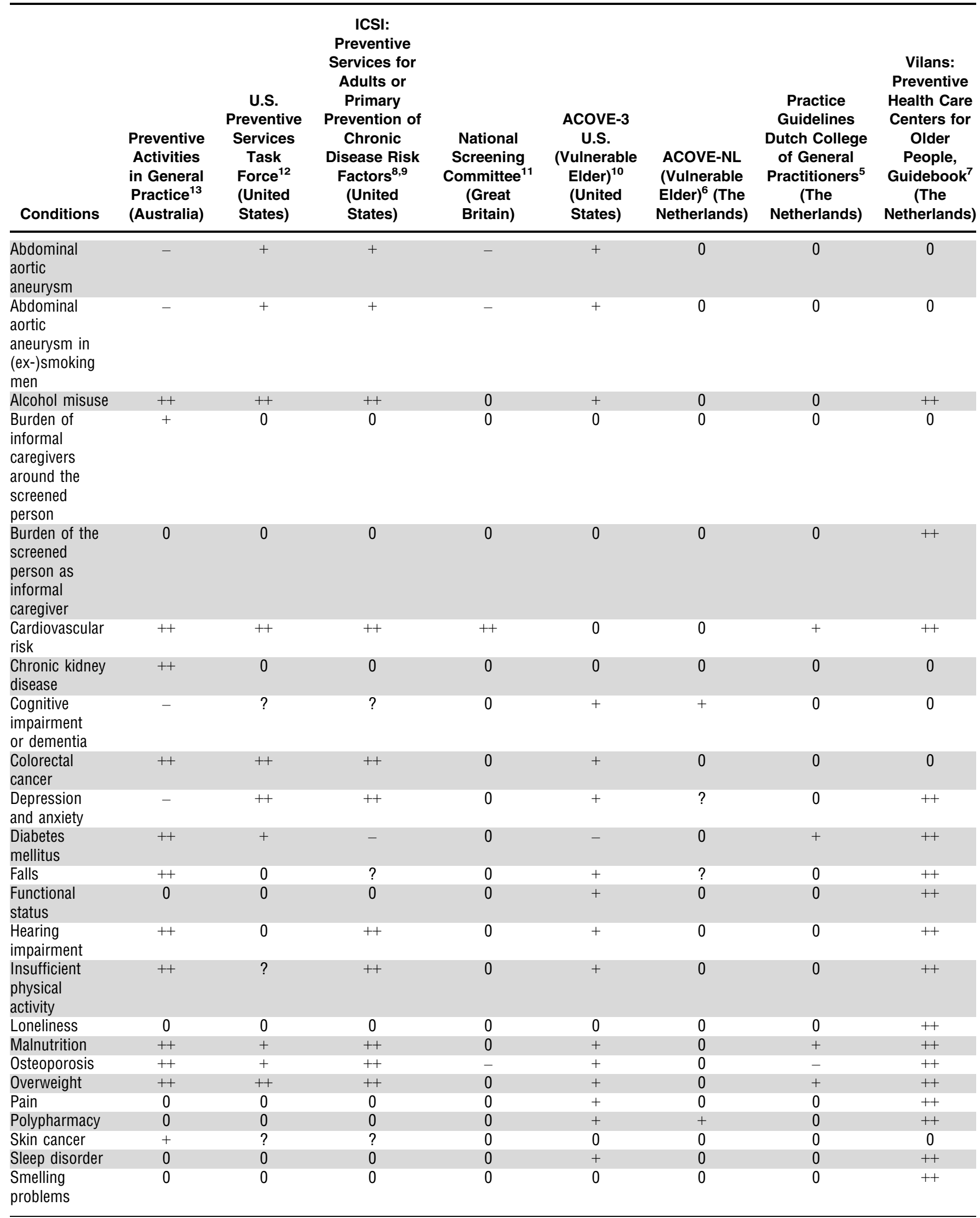


Table 2 (Contd.)

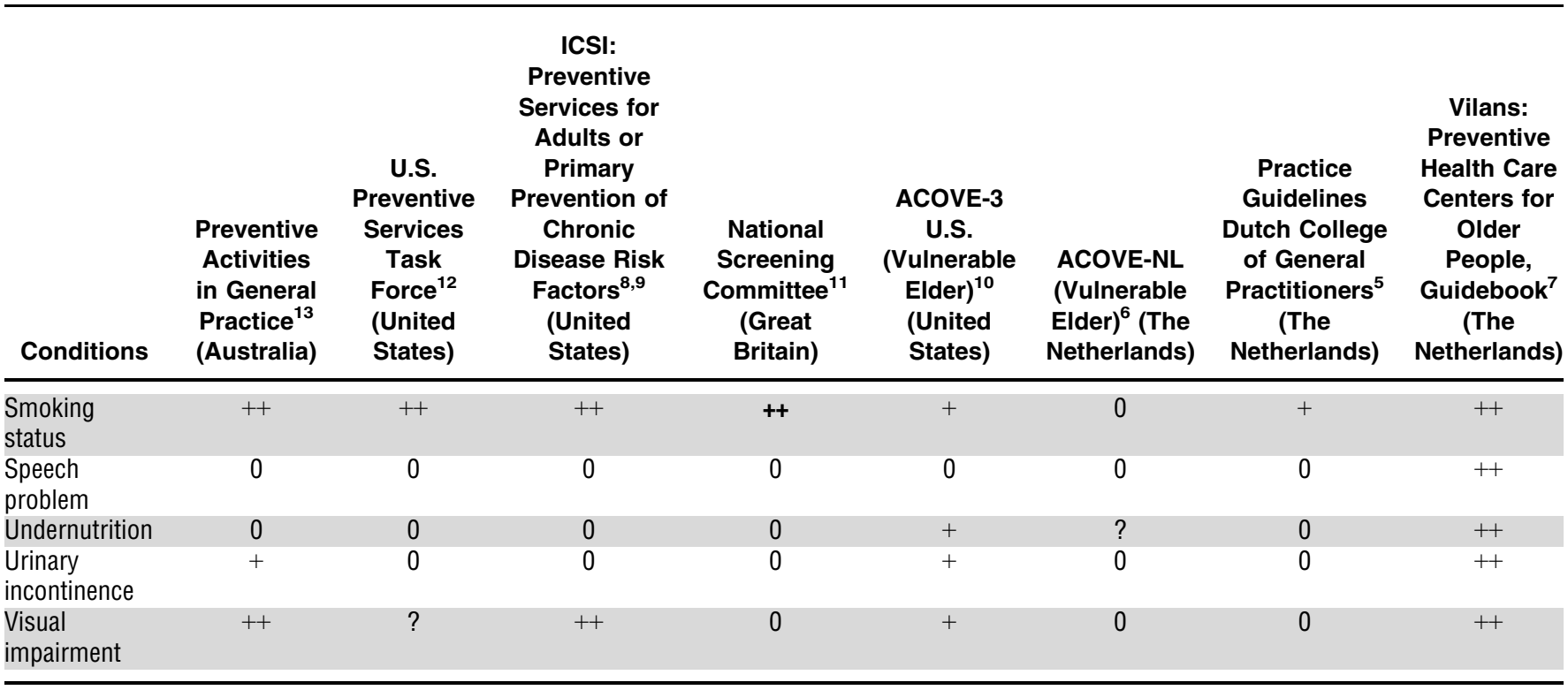

$++=$ screening for older people recommended; $+=$ screening for older people at risk recommended (including vulnerable elderly); $-=$ advice against screening; ? = insufficient evidence for or against; 0 = screening not a topic.

ACOVE $=$ Assessing Care of the Vulnerable Elders.

screening and screening for smoking status were rated appropriate in the vital population aged 60 to 74 . Uncertainty (median range: 4-6 or disagreement) about the appropriateness was rated in $24 \%(63 / 261)$ of the scores. Of all uncertain outcomes, $67 \%$ concerned the vulnerable population. The panelists argued that lack of sufficient evidence to fulfill the criteria of Wilson and Jungner is mainly due to lack of research in this population as such. Based on their expertise in clinical practice, they assumed that development of specific tests and interventions for this group may generate evidence and will lead to benefits of screening, especially when the screening approach is embedded in regular care.

\section{Influence of Age}

In contrast to expectations, the age category of the persons did not strongly influence the ratings of the panel (Table 3). Exceptions to this were cardiovascular screening and smoking status (influence of age in all three groups of health status); abdominal aortic aneurysm in (ex-)smoking men, colorectal cancer, burden of the screened person as informal caregiver (influence of age in the general and vital population); and urinary incontinence (influence of age in the vital population). For cardiovascular screening of older people, the main problem is lack of a suitable test. The panelists considered that Framingham Study scores were not valid for the older age categories because these scores do not predict cardiovascular mortality in the oldest old. ${ }^{33}$ For smoking, abdominal aortic aneurysm in (ex-)smoking men, and colorectal cancer, the importance of screening declines with increasing age for different reasons (e.g., for smoking, there is insufficient evidence for the yield of stopping at older age; for aneurysm, the risk of a surgical procedure increases with age; and for colorectal cancer, the natural history at older age is unknown, and the risk of surgery increases with age). In contrast, the appropriate- ness of screening for urinary incontinence and for the burden of the screened person as informal caregiver increases with age, mainly because the yield increases.

\section{Reasons for Uncertainty and Inappropriateness}

When the panelists expected benefits of screening according to their expert opinion, although evidence was lacking, they rated the condition in the uncertain range. Screening for a condition was rated in the inappropriate range when evidence from literature was against screening or when evidence was lacking and the panelists expected no benefit according to their expert opinion. In the panel discussions, the most frequently used argument for inappropriateness was lack of evidence for effective interventions (Wilson and Jungner criterion 2). ${ }^{23}$ There was sometimes a perceived lack of a rational evidence-based intervention (e.g., dementia, smelling problems), and sometimes it was assumed that adherence to advice or treatment after a positive screening would be too low on the basis of experience or circumstantial scientific evidence (e.g., urinary incontinence, hearing aid, alcohol abuse). Furthermore, the panel thought some conditions to be of insufficient importance (Wilson and Jungner criterion 1) because the prevalence was too low to warrant screening (e.g., skin cancer in the Netherlands) or the relevance of screening for the condition was not considered to be high enough (e.g., pain and sleeping disorders). In general, the panelists expected that people with these problems and motivation for subsequent interventions would already be seeking help. For some conditions, a suitable test or examination was lacking (Wilson and Jungner criterion 5): too many false positives (fecal occult blood test for colorectal cancer) or too many false negatives (alcohol abuse, osteoporosis), problems with acceptance of the test (colonoscopy), or test not validated for screening (De Jong-Gierveld Loneliness Scale). ${ }^{34}$ 
Table 3. Appropriateness of Screening to Prevent Functional Decline in the General Older Population, Vital Older Persons, and Vulnerable Older Persons, Stratified According to Age

Final Rating, Median ${ }^{\dagger}$

\begin{tabular}{|c|c|c|c|c|c|c|c|c|c|}
\hline \multirow{4}{*}{ Conditions to Screen For ${ }^{\star}$} & \\
\hline & \multirow{2}{*}{\multicolumn{3}{|c|}{$\begin{array}{l}\text { General Older } \\
\text { Population }\end{array}$}} & \multicolumn{6}{|c|}{ Older Persons } \\
\hline & & & & \multicolumn{3}{|c|}{ Vital } & \multicolumn{3}{|c|}{ Vulnerable } \\
\hline & $60-74$ & $75-84$ & $\geq 85$ & $60-74$ & $75-84$ & $\geq 85$ & $60-74$ & $75-84$ & $\geq 85$ \\
\hline \multicolumn{10}{|l|}{ At least one rating appropriate } \\
\hline Insufficient physical activity & 7 & 7 & 7 & 7 & 7 & 7 & 7 & 7 & 7 \\
\hline \multicolumn{10}{|l|}{ At least one rating uncertain } \\
\hline Burden of the screened person as informal caregiver & 3 & 4 & 4 & 3 & 4 & 4 & 5 & 5 & 5 \\
\hline Hearing impairment & 4 & 4 & 4 & 2 & 2 & 2 & 5 & 5 & 5 \\
\hline Urinary incontinence & 3 & 3 & 3 & 3 & 4 & 4 & 5 & 5 & 5 \\
\hline Colorectal cancer & 5 & 4 & 2 & 6 & 5 & 2 & 3 & 3 & 2 \\
\hline Burden of informal caregivers around the screened person & 1 & 2 & 2 & 1 & 1 & 2 & $5 D$ & $5 \mathrm{D}$ & $5 \mathrm{D}$ \\
\hline Malnutrition & 2 & 2 & 2 & 2 & 2 & 2 & 4D & $4 D$ & $4 \mathrm{D}$ \\
\hline Pain & 2 & 2 & 2 & 1 & 1 & 1 & 5 & 5 & 5 \\
\hline Polypharmacy & 2 & 2 & 2 & 2 & 2 & 2 & 5 & 5 & 5 \\
\hline Undernutrition & 3 & 3 & 3 & 2 & 2 & 2 & 5 & 5 & 5 \\
\hline Visual impairment & 2 & 2 & 2 & 2 & 2 & 2 & 6 & 6 & 6 \\
\hline Abdominal aortic aneurysm in (ex-)smoking men & 4 & 2 & 2 & 5 & 3 & 2 & 2 & 2 & 1 \\
\hline \multicolumn{10}{|l|}{ All ratings inappropriate } \\
\hline Abdominal aortic aneurysm & 2 & 1 & 1 & 2 & 1 & 1 & 1 & 1 & 1 \\
\hline Alcohol misuse & 2 & 2 & 2 & 2 & 2 & 2 & 2 & 2 & 2 \\
\hline Chronic kidney disease & 2 & 2 & 2 & 2 & 2 & 2 & 3 & 3 & 3 \\
\hline Diabetes mellitus & 3 & 3 & 2 & 3 & 3 & 2 & 2 & 2 & 2 \\
\hline Falls & 2 & 2 & 2 & 2 & 2 & 2 & 3 & 3 & 3 \\
\hline
\end{tabular}

Ranked according to appropriateness and alphabetically.

${ }^{\dagger}$ Range: 1-3, inappropriate; range: 4-6, uncertain; range: 7-9, appropriate.

$\mathrm{D}=$ disagreement: at least three panelists rated in the 1-3 range and at least three panelists rated in the 7-9 range.

\section{DISCUSSION}

\section{Principle Findings}

Despite increasing interest in screening of communitydwelling older people and the recommendations in guidelines, the Dutch panel considered screening of only a few conditions to be appropriate. Screening for insufficient physical activity to prevent functional decline is appropriate for all older persons. Screening for cardiovascular risk factors and smoking status are considered useful for the general older population aged 60 to 74 but not for vulnerable older people in the same age range. There is insufficient evidence to support screening for the other investigated conditions.

During the face-to-face meeting, the experts emphasized that an uncertain or inappropriate rating does not mean that the condition is irrelevant but that there was insufficient evidence to recommend an active screening approach. To conclude that screening contributes to the prevention of functional decline, screening must at least approximately meet the criteria of Wilson and Jungner. When evidence to fulfill the criteria of Wilson and Jungner was lacking or inconclusive, the experts' opinions about a potential benefit to prevent functional decline were taken into account. It was not thought that strong evidence supported interventions that merely stimulate well-being (e.g., interventions to address loneliness), although based on experience, the panelists expected at least some benefit from these interventions.

Vulnerability was considered to be an important factor in the determination of appropriateness of screening. For 11 of the 29 conditions, the panelists were uncertain about the appropriateness of screening vulnerable older people, whereas they considered screening of older persons with good vitality for the same condition to be 
inappropriate. Because of lack of research data on the vulnerable group, the panelists had to rely on their expert opinion to rate these screening options. They expect benefit from screening when more tests and interventions are developed for this group. Because the majority of vulnerable older people already receive medical care for their chronic disease(s), the panelists expected more benefit from improving regular care than from a separate screening program.

Age played a small role during the panel discussions. Appropriateness of screening was modified according to age for only six conditions: smoking status, cardiovascular risk, abdominal aortic aneurysm in (ex-)smoking men, colorectal cancer, burden of the screened person as informal caregiver, and urinary incontinence. A possible explanation for this is the relationship between age and vulnerability, with the latter being the discriminating factor in rating.

Some guidelines ${ }^{5,7,11,13}$ claim that their recommendations are based on the criteria of Wilson and Jungner, although there are marked differences between the recommendations in these guidelines. A possible explanation for the differences in these guidelines is a difference in the validity of the guideline procedures. For example, the Vilans guidebook, ${ }^{7}$ which contains the most positive advice, is a descriptive protocol of available screening conditions for older people rather than an evidence-based screening guideline. Also, considerable differences may exist between countries in the interpretation of evidence because of cultural differences and differences in healthcare systems, which influence recommendations in the guidelines. ${ }^{35}$ The validity of the guideline processes (e.g., using the Appraisal of Guidelines, Research, and Evaluation in Europe (AGREE) instrument) ${ }^{36}$ was not formally assessed in the present study, because the main focus was determination of the appropriateness of screening by the expert panel.

Comparison of the outcome of the RAND/UCLA appropriateness method with the recommendations of the various guidelines shows considerable differences between guidelines. The panel rating was more in accordance with the European guidelines than with the U.S. and Australian guidelines, probably because of an underlying cultural difference; (e.g., when evidence is lacking, Dutch healthcare professionals tend to rely on the adage primum non nocere, to defend patients from iatrogenic harm). Vulnerable older people are at higher risk for expected and unexpected side effects of confirmatory testing that follow a screening test and subsequent treatment. ${ }^{4}$ In addition, organization of care and healthcare availability may play a role; all inhabitants in the Netherlands have healthcare insurance, and almost everyone is registered with one general practice over many years. People aged 75 and older contact their general practitioner more than 16 times a year, ${ }^{37}$ which often allows the general practitioner to detect relevant changes in and problems with the aging process on a personal level.

Osteoporosis, for example, is a condition in which these cultural and healthcare differences played a role in the panelists' discussions. For osteoporosis, earlier research resulted in evidence-based methods to identify risk for osteoporotic fractures and effective medications to reduce fractures, but as the U.S. Preventive Services Task Force showed in its review of July $2010,{ }^{38}$ no trials have directly evaluated screening effectiveness, harms, and intervals between screening. This lack of direct evidence leaves room for weighing and interpretation, apparently resulting in the overall finding that European guidelines contained negative advice to screen for osteoporosis, whereas the non-European guidelines recommended screening. In the present study, the panelists considered that, in the Netherlands, assessment of osteoporosis was already part of treatment in older people after fracture. In people using corticosteroids for a prolonged period, prevention and treatment of osteoporosis also form part of the therapeutic plan. This means that the high-risk groups are already assessed in the context of "normal" care. Only older people without a fracture and without use of corticosteroids are still unscreened. For this low-risk group, the panelists argued that, although screening for osteoporosis in general has not been proven to be effective, screening in this remaining low-risk group will be even less effective. Therefore, according to the panelists, there is insufficient evidence to support screening for osteoporosis, especially regarding the screening test (too many false negatives in this low-risk group; Wilson and Jungner criterion 5).

\section{Strengths and Limitations}

The present study has several strengths. First, the focus on healthy aging by preventing functional decline is relatively new in studies on screening. In this study, the objective of screening older people was not primarily to prevent and postpone disease and death but rather to support the ability of older people to function as independently as possible. ${ }^{18}$ The results of the study indicate the need for more high-quality studies to support the benefit of screening to prevent functional decline. Another strength is the multidisciplinary panel, because the composition of the panel is known to influence the outcome of the RAND/UCLA appropriateness method. ${ }^{39}$ Most users of the RAND/UCLA method recommend using a multidisciplinary panel to better reflect the variety of specialties involved in decisions on treatment. ${ }^{22}$ If another panel in which the composition in terms of disciplines is maintained repeats the same procedure, the results will be reproduced with a high level of agreement. ${ }^{27,30,40}$ In the present study, the initial disagreement in the first round $(23 \%)$ meant that the panel composition adequately reflected the different opinions about screening in health care. During the discussion, all panelists were engaged in a positive-critical way and were willing to change their opinion, if necessary.

One limitation of the present study is the specific Dutch context in which the RAND/UCLA appropriateness method was used; this might influence generalizability. It would be interesting if panels in other countries would replicate this study in order to compare the findings.

Another limitation is that it was not feasible to perform exhaustive systematic reviews for all 29 conditions for all 10 criteria of Wilson and Jungner. Instead, the literature on which the guidelines were based was collected, and experts were invited to complete and update these files with recent literature. This practical approach is in accordance with the RAND/UCLA appropriateness method. ${ }^{22}$ 


\section{Clinical Implications and Future Research}

The results of the RAND/UCLA appropriateness method indicate that, according to the panelists, only screening of the general older population for insufficient physical activity, smoking status, and cardiovascular risk in specific groups is recommended to prevent functional decline. The uncertain or inappropriate rating of the remaining screening conditions does not mean that the conditions are not relevant but that there is insufficient evidence to recommend an active screening approach at the population level. For the conditions rated uncertain, mostly regarding the vulnerable older population, evidence was lacking, although based on their clinical experience, the panelists expected potential benefit from screening embedded in the regular care for this group of older people. It is important in future research to detect effective screening approaches and subsequent treatments to maintain functional status and related quality of life for this group. Then, screening and monitoring as part of regular care will support healthier aging by preventing or delaying functional decline.

\section{ACKNOWLEDGMENTS}

The authors thank DJA van Dijk-van Dijk, MSc, Leiden University Medical Center and BJC Middelkoop, MD, $\mathrm{PhD}$, Leiden University Medical Center, for general advice and all experts for their literature review and for supplying additional evidence: $\mathrm{T}$ Antheunissen, MD, GGZ Leiden (alcohol misuse), JJ van Binsbergen, MD, PhD, Radboud University Nijmegen Medical Centre (nutrition and overweight), $\mathrm{NH}$ Chavannes, $\mathrm{MD}, \mathrm{PhD}$, Leiden University Medical Center (smoking status), Y Groeneveld, MD, $\mathrm{PhD}$, Leiden University Medical Center (diabetes), JF Hamming, MD, PhD, Leiden University Medical Center (abdominal aortic aneurysm), $\mathrm{M}$ Hopman-Rock, PhD, MA, MSc, TNO Quality of Life, Leiden and VU University Medical Center, Amsterdam (falls and insufficient physical activity), J de Jong Gierveld, PhD, Netherlands Interdisciplinary Demographic Institute (loneliness and social support), GIJM Kempen, PhD, CAPHRI School for Public Health and Primary Care, Maastricht University (functional status), A Knuistingh Neven, MD, PhD, Leiden University Medical Center (sleep disorders), RM Kok, $\mathrm{MD}, \mathrm{PhD}$, Parnassia Psychiatric Institute (depression and anxiety), JAPM de Laat, PhD, Leiden University Medical Center (hearing impairment), A Lagro-Jansen, $\mathrm{MD}, \mathrm{PhD}$, Radboud University Nijmegen Medical Centre (urinary incontinence), P Lips, MD, PhD, VU University Medical Center (osteoporosis), F Verhey, MD, PhD, School for Mental Health and Neuroscience/Alzheimer Centre Limburg and Maastricht University Medical Center (cognitive impairment), LJ van Rijn, MD, PhD, VU University Medical Center (visual impairment), AHP Niggebrugge, MD, $\mathrm{PhD}$, Bronovo Hospital (colorectal cancer and skin cancer), W de Ruijter, MD, PhD, Leiden University Medical Center (cardiovascular risk), YWJ Sijpkens, MD, PhD, Leiden University Medical Center (chronic kidney disease), PAGM de Smet, PharmD, PhD, Radboud University Nijmegen Medical Centre (polypharmacy), MM Verduijn, MSc, Dutch College of General Practitioners (pain) and G Visser, MA, Vilans, Centre of Expertise for long-term care (burden of the informal caregiver, smelling problems, social well-being, spare time, and speech problems). These experts received 150 Euro each as compensation for their contributions.

Conflict of Interest: R van Overbeek is an employee of Knowledge Centre Vilans, whose guideline was used. The honorarium panelists received 1,000 Euro and the honorarium moderator received 2,000 Euro.

This study was partly funded by the Organization for Health Research and Development, the Netherlands.

Author Contributions: Drewes had full access to all of the data in the study and takes responsibility for the integrity of the data and the accuracy of the data analysis. Drewes, Gussekloo, van der Meer, Assendelft: Study concept and design. Drewes, Gussekloo, van der Meer, Rigter, Dekker, Goumans, Metsemakers, van Overbeek, de Rooij, Schers, Schuurmans, Sturmans, de Vries, Westendorp, Wind, Assendelft: Analysis and interpretation of data. Drewes: Drafting of the manuscript. Gussekloo, van der Meer, Rigter, Dekker, Goumans, Metsemakers, van Overbeek, de Rooij, Schers, Schuurmans, Sturmans, de Vries, Westendorp, Wind, Assendelft: Critical revision of the manuscript for important intellectual content.

Sponsor's Role: The sponsor had no role in the design or conduct of the study; collection, management, analysis, or interpretation of the data; or preparation, review, or approval of the manuscript.

\section{REFERENCES}

1. Klein WC, Bloom M. Successful Aging: Strategies for Healthy Living. New York: Plenum Press, 1997.

2. Infeld DL, Whitelaw N. Policy initiatives to promote healthy aging. Clin Geriatr Med 2002;18:627-642.

3. Oliver M. Let's not turn elderly people into patients. BMJ 2009;338:b873.

4. Clarfield AM. Screening in frail older people: An ounce of prevention or a pound of trouble? J Am Geriatr Soc 2010;58:2016-2021.

5. Dutch College of General Practitioners. [Practice Guidelines from the Dutch College of General Practitioners 2009]. Utrecht: Dutch College of General Practitioners, 2008.

6. van der Ploeg E, Depla MF, Shekelle P et al. Developing quality indicators for general practice care for vulnerable elders: Transfer from US to the Netherlands. Qual Saf Health Care 2008;17:291-295.

7. Bakker E, Jaspers N, Kraakman M et al. [Preventive Health Care Centers for Older People, Guidebook]. Utrecht: Vilans, the Knowledge Centre on Ageing, 2008.

8. Institute for Clinical Systems Improvement. Preventive Services for Adults, 14th Ed. Bloomington, MN: Institute for Clinical Systems Improvement, 2008.

9. Institute for Clinical Systems Improvement. Primary Prevention of Chronic Disease Risk Factors, 1st Ed. Bloomington, MN: Institute for Clinical Systems Improvement, 2008.

10. RAND Corporation. Assessing the Care of Vulnerable Elders, Potential Quality Indicators and Literature Review, ACOVE-3. Santa Monica, CA: RAND Corporation, 2006.

11. UK National Screening Committee. Second Report of the UK National Screening Committee. London: Department of Health, 2000.

12. Agency of Healthcare Research and Quality. The Guide to Clinical Preventive Services 2008, Recommendations of the U.S. Preventive Services Task Force. Rockville, MD: Agency of Healthcare Research and Quality, 2008.

13. The Royal Australian College of General Practitioners. Guidelines for Preventive Activities in General Practice, 6th Ed. South Melbourne: The Royal Australian College of General Practitioners, 2005.

14. Swedish National Institute of Public Health. Healthy Ageing: A Challenge for Europe. Stockholm: Swedish National Institute of Public Health, 2007.

15. Division of Ageing and Seniors, Public Health Agency of Canada. Healthy Aging in Canada: A New Vision, a Vital Investment. from Evidence to Action. A background paper for the Federal, Provincial and Territorial 
Committee of Officials (Seniors). Ottawa: Division of Aging and Seniors, Public Health Agency of Canada, 2006.

16. National Center for Chronic Disease Prevention and Health Promotion. Healthy Aging: Improving and Extending Quality of Life Among Older Americans, at a Glance 2010. Atlanta, GA: National Center for Chronic Disease Prevention and Health Promotion, 2010.

17. Prime Minister's Science Engineering and Innovation Council. Promoting Healthy Ageing in Australia. Canberra: Prime Minister's Science Engineering and Innovation Council. 2003.

18. Health Council of the Netherlands. [Prevention in the Elderly: Focus on Functioning in Daily Life], 2009/07 Ed. The Hague: Health Council of the Netherlands, 2009.

19. Holland WW, Stewart S, Masseria C. Policy Brief: Screening in Europe. Brussels: World Health Organization, on behalf of the European Observatory on Health Systems and Policies, 2006.

20. Brook RH, Chassin MR, Fink A et al. A method for the detailed assessment of the appropriateness of medical technologies. Int J Technol Assess Health Care 1986;2:53-63.

21. Brook RH. Assessing the appropriateness of care-Its time has come. JAMA 2009;302:997-998.

22. Fitch K, Bernstein SJ, Aguilar MD et al. The RAND/UCLA Appropriateness Method User's Manual. Santa Monica, CA: RAND, 2001.

23. Wilson JMG, Jungner G. Principles and Practice of Screening for Disease. Geneva: World Health Organization, 1968.

24. Depp CA, Jeste DV. Definitions and predictors of successful aging: A comprehensive review of larger quantitative studies. Am J Geriatr Psychiatry 2006;14:6-20.

25. Manton KG. Recent declines in chronic disability in the elderly U.S. population: Risk factors and future dynamics. Annu Rev Public Health 2008;29:91-113.

26. Rockwood K, Fox RA, Stolee P et al. Frailty in elderly people: An evolving concept. Can Med Assoc J 1994;150:489-495.

27. Shekelle PG, Kahan JP, Bernstein SJ et al. The reproducibility of a method to identify the overuse and underuse of medical procedures. N Engl J Med 1998;338:1888-1895.

28. Hemingway H, Crook AM, Feder G et al. Underuse of coronary revascularization procedures in patients considered appropriate candidates for revascularization. N Engl J Med 2001;344:645-654.

29. Merrick NJ, Fink A, Park RE et al. Derivation of clinical indications for carotid endarterectomy by an expert panel. Am J Public Health 1987;77:187-190.

30. Shekelle P. The appropriateness method. Med Decis Making 2004;24:228 231.

31. Rigter H, Meijler AP, McDonnell J et al. Indications for coronary revascularisation: A Dutch perspective. Heart 1997;77:211-218.
32. McGory ML, Shekelle PG, Ko CY. Development of quality indicators for patients undergoing colorectal cancer surgery. J Natl Cancer Inst 2006;98:1623-1633

33. de Ruijter W, Westendorp RG, Assendelft WJ et al. Use of Framingham risk score and new biomarkers to predict cardiovascular mortality in older people: Population based observational cohort study. BMJ 2009;338:a3083.

34. Jong-Gierveld J, Kamphuis F. The development of a Rasch-type loneliness scale. Applied Psychol Measure 1985;9:289-299.

35. Burgers JS, Grol R, Klazinga NS et al. Towards evidence-based clinica practice: An international survey of 18 clinical guideline programs. Int J Qual Health Care 2003;15:31-45.

36. AGREE Collaboration. Development and validation of an international appraisal instrument for assessing the quality of clinical practice guidelines: The AGREE project. Qual Saf Health Care 2003;12:18-23.

37. Cardol M, van Dijk L, de Jong JD et al. [Second National Study into Diseases and Activities in General Practice. Primary Care: What Does the Gatekeeper Do?]. Utrecht/Bilthoven: NIVEL/RIVM, 2004.

38. Nelson HD, Haney EM, Dana T et al. Screening for osteoporosis: An update for the U.S. Preventive Services Task Force. Ann Intern Med 2010;153:99-111.

39. Kahan JP, Park RE, Leape LL et al. Variations by specialty in physician ratings of the appropriateness and necessity of indications for procedures. Med Care 1996;34:512-523

40. Tobacman JK, Scott IU, Cyphert S et al. Reproducibility of measures of overuse of cataract surgery by three physician panels. Med Care 1999;37:937-945.

\section{SUPPORTING INFORMATION}

Additional Supporting Information may be found in the online version of this article:

Appendix S1. Overview of the RAND/UCLA appropriateness method.

Please note: Wiley-Blackwell is not responsible for the content or functionality of any supporting materials supplied by the authors. Any queries (other than missing material) should be directed to the corresponding author for the article. 